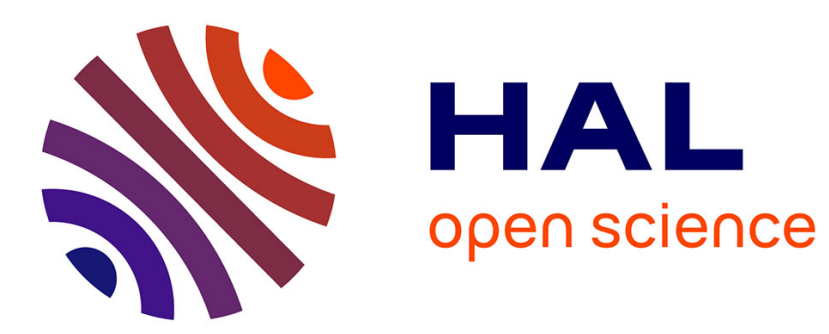

\title{
A micromechanics-based elastoplastic damage model for quasi-brittle rocks
}

\author{
N. Xie, Q.-Z. Zhu, L.-H. Xu, J. F. Shao
}

\section{To cite this version:}

N. Xie, Q.-Z. Zhu, L.-H. Xu, J. F. Shao. A micromechanics-based elastoplastic damage model for quasi-brittle rocks. Computers and Geotechnics, 2011, 38 (8), pp.970-977. hal-00749199

\section{HAL Id: hal-00749199 \\ https://hal.science/hal-00749199}

Submitted on 6 Nov 2012

HAL is a multi-disciplinary open access archive for the deposit and dissemination of scientific research documents, whether they are published or not. The documents may come from teaching and research institutions in France or abroad, or from public or private research centers.
L'archive ouverte pluridisciplinaire HAL, est destinée au dépôt et à la diffusion de documents scientifiques de niveau recherche, publiés ou non, émanant des établissements d'enseignement et de recherche français ou étrangers, des laboratoires publics ou privés. 


\title{
A micromechanics-based elastoplastic damage model for quasi-brittle rocks
}

\author{
N. Xie ${ }^{\mathrm{a}, \mathrm{b}}$, Q.Z. Zhu ${ }^{\mathrm{c}}$, L.H. Xu ${ }^{\mathrm{a}}$, J.F. Shao ${ }^{\mathrm{b}, *}$, \\ a School of Civil Engineering, Wuhan University, Wuhan 430072, China \\ ${ }^{\mathrm{b}}$ Laboratoire de Mécanique de Lille (UMR8107 CNRS), Université de Lille I, \\ Cité Scientifique,59655 Villeneuve d'Ascq, France \\ ${ }^{\mathrm{c}}$ Laboratoire Modélisation et Simulation Multi-Echelle (UMR8208 CNRS), Université de Paris-EST, \\ Boulevard Descartes, F-77454, Marne la Valée Cedex-2, France \\ ${ }^{*}$ Corresponding author
}

\begin{abstract}
This paper presents a micromechanics-based elastoplastic damage model for quasi-brittle rocks in compression stress state. The plastic strain is considered to be related to frictional sliding along microcracks and inherently coupled with damage evolution. By following a homogenization procedure, we first determine the free energy for the matrix-cracks system. The thermodynamic force associated with the inelastic strain contains a back stress which controls material hardening. Next, a Coulomb-type friction criterion serving as plastic yielding function and a strain energy release rate based damage criterion are proposed for determination of plastic flow and crack propagation. These micro-macro thermodynamic formulations allow to reducing significantly model's parameters with respect to phenomenological models. The proposed model is applied to simulate triaxial compression tests on two sets of diabase samples, the first cored from a fresh diabase rock mass and the second from a slightly weathered one. Comparisons between numerical predictions and test data are presented.
\end{abstract}

Key words: Thermodynamics; homogenization; isotropic damage; friction; microcracks; semi-brittle rocks.

Email addresses: qizhi.zhu@gmail.com (Q.Z. Zhu), jianfu.shao@univ-lille1.fr (J.F. Shao).

Preprint submitted to Elsevier Science

February 15, 2011 


\section{Introduction}

The Danjiangkou Water Reservoir, located in central China, is one of the most important water conservancy projects in China. As the water source of the South-to-north water diversion project, it provides water to the naturally water deficient area in north China. Since it began to store water in 1970s, lots of seismic activities were monitored, with a maximum earthquake magnitude of 4.7 recorded in 1973. To make sure the stability and security of the water reservoir, it is pre-requisite to have sufficient recognition of the mechanical behavior of the rocks that formed the base of the reservoir.

Various kinds of rocks are found in the area of Danjiangkou Water Reservoir, such as diabase rock, sandstone, schist rock, etc. Among them, diabase is one of the most widely distributed. Hence, in this paper, we choose to study the mechanical behavior of diabase rock samples to provide useful data for the stability analysis of the reservoir.

As a typical brittle material, the mechanical behavior of diabase rock is characterized by high peak strength, small deformation, scarce residual strength and strong pressure sensitivity. For brittle materials such as rocks and concrete, it is widely accepted that the nucleation, growth and coalescence of microcracks are the main causes of the mechanical deterioration of the materials leading to failure (Brace and Bombolakis, 1963; Wawersik and Brace, 1971; Wong, 1982; Kranz, 1983; Horii and Nemat-Nasser, 1985). To this end, the concept of damage has been introduced to describe the material degradation and the mechanical response of such quasi brittle materials (Chow and June, 1987; Ju, 1989; Chaboche and P., 2005; Murakami and Kamiya, 1996; Halm and Dragon, 1996). Generally, there are two families of constitutive models describing the stress-strain relationship with damage. The first one is phenomenological models and the other consists of the models based on micromechanical approaches. In the phenomenological models, the free energy of the material is interpreted as a function of a series of internal variables, such as plastic strain, damage, etc., with a scalar variable representing isotropic damage or a second or fourth rank tensor representing anisotropic one. This kind of models can describe the main mechanical characters of brittle materials to a certain extend. However, these models are generally based on some viewable phenomena and experiences rather than solid mathematical derivation. Hence, there exist usually excessive parameters to be determined in these models, and many of them have no definite physical significance. On the other hand, in recent years, many researches proposed to use micromechanical approaches to deal with damage problem in brittle materials (Andrieux et al., 1986; Gambarotta and Lagomarsino, 1993; Pensee et al., 2002; Dormieux et al., 2006; Zhu et al., 2008a,b). In such models, the stress-strain relationship is first determined at relevant material scales, and then a homogenization procedure is used to link the microscopic local stresses and strains to the macroscopic ones, leading finally to macroscopic 
behaviors of the material. Meanwhile, proper criteria for microcrack propagation and damage growth should be proposed. Therefore, the micromechanical models are able to account for physical mechanisms involved in damage process. These models have generally much less parameters compared with the phenomenological ones, and many important factors, such as the unilateral effects due to opening-closure of the microcracks, coupling between friction and damage on cracks faces, as well as interaction between cracks can be taken into account in these models.

In this paper, based on experimental observations upon the diabase rocks, an improved micromechanicsbased isotropic elastoplastic damage model is developed, with coupling between friction and damage taken into consideration. Damage is assumed to be isotropic for the sake of simplicity and the emphasis is put on the coupling between damage and friciton. Triaxial compression test results of two groups of diabase samples, fresh and slightly weathered ones, are shown respectively. Using the micromechanical model, predictions of mechanical responses for both groups of samples are performed and compared with the testing data. The weathering effect on mechanical behavior of diabase rock is detailed with some remarking analysis.

\section{Description of material and testing results}

Triaxial compression tests were performed on two sets of diabase samples. One set is cored from a fresh rock mass, and the other from a slightly weathered one. Both the two rock masses were drilled from Danjiangkou water reservoir, the base of which consists of rocks of Presinian Period mainly. After extraction on site, the rock masses were stored in a sealed container to keep the natural water content.

The dimension of the testing samples is $\$ 50 \mathrm{~mm} \times 100 \mathrm{~mm}$. Other tests were also carried out in order to collect the different physical characteristics between the fresh samples and the slightly weathered ones. The results are shown in Table 1. The porosity and bulk density of rock samples were determined by the saturation-buoyancy techniques recommended by ISRM. Other parameters are also obtained by the standard methods recommended in ISRM.

Visually, the slightly weathered sample has a darker colour compared with the fresh one, and there are a few weathering fissures in it. In Table 1, we can see that the dry density and specific gravity of these two rocks are quite close, indicating the weathering extent is moderate (Bozkurtoglu et al., 2006), and the cement structures of these two materials are basically unchanged. The most important difference between the fresh samples and the slightly weathered ones is the difference in porosity (see Table 1), which has important influences on the mechanical behaviour of the samples, as will be shown in the following. 
Table 1

Comparison of physical parameters of two sets of diabase rock samples

\begin{tabular}{cccc}
\hline \hline Samples & Dry density $\left(\mathrm{g} / \mathrm{cm}^{3}\right)$ & Water content (\%) & Porosity (\%) \\
\hline Fresh diabase & 2.97 & 0.059 & 0.37 \\
& & & Specific gravity \\
Slightly weathered diabase & 2.91 & 0.59 & 2.69 \\
\hline
\end{tabular}

The triaxial compression tests were carried out on MTS (material testing system), a testing system for various materials. In particular, this equipment is famous for its great rigidity and the ability to provide high pressure. Therefore it is very suitable for testing rock samples.

Testing results for both two sets are shown in the figures 1 and 2. It can be seen that for fresh rock samples (Fig. 1), the stress-strain curves show obvious brittleness, the nonlinear deformation before peak strength is fairly small, and that the resistance drops dramatically afterwards. Besides, when confining pressure increases to $40 \mathrm{MPa}$, the stress-strain curve still exhibits obvious brittleness. There seems no transition from brittleness to ductility as confining pressure increases, which is mainly due to the quite small porosity of the samples.

For the slightly weathered rock samples (see Fig. 2), the stress-strain curves also show obvious brittleness. But when confining pressure gets to $20 \mathrm{MPa}$, the nonlinear deformation before peak strength becomes obvious, which is because of the relatively greater porosity and initial cracks compared with the fresh samples. On the other hand, significant reduction in peak strength can be found between fresh samples and slightly weathered ones. Although the amount of mineral alteration is small, the strength of the slightly weathered rock decreases due to the augmentation of porosity in the form of microcracks. Similar conclusions can be found in Tugrul (2004).

\section{Formulation of the constitutive model}

Consider a representative elementary volume (r.e.v.), occupying geometric domain $\Omega$ (boundary $\partial \Omega$ ) and composed of the solid matrix with the elasticity tensor $\mathbb{C}^{s}$ and penny-shaped microcracks randomly distributed in the matrix. To make direct use of the Eshelby's solution, all microcracks are approximated as flat ellipsoids. Geometrically, such cracks can be identified by the unit vector $\underline{n}$ normal to crack plane and characterized via the aspect ratio $\frac{c}{a}$, noted $\epsilon$, with $a$ and $c$ being the radius of the circular crack and 
the half-length of the small axis, respectively. The volume fraction $\phi^{r}$ of the $r^{\text {th }}$ family of microcracks of normal $\underline{n}^{r}$ then reads

$$
\phi^{r}=\frac{4}{3} \pi a_{r}^{2} c_{r} \mathcal{N}_{r}=\frac{4}{3} \pi \epsilon d^{r},
$$

where $\mathcal{N}_{r}$ denotes the crack density (number of cracks per unit volume) of the family in consideration, and $d^{r}=\mathcal{N}_{r} a_{r}^{3}$ is defined as crack damage parameter (Budiansky and O'Connel, 1976) and serves as internal variables in micromechanical analysis.

Under loading, the total strain $\boldsymbol{E}$ in the cracked medium is attributed by two sources: the first, $\boldsymbol{E}^{m}$, related to the elastic solid matrix and the second, denoted by $\boldsymbol{E}^{c}$, due to displacement discontinuities between crack lips. By defining $\boldsymbol{E}$ as the integration of the strain field $\varepsilon(\boldsymbol{x})$ over the above-described elementary volume, i.e. $\boldsymbol{E}=\frac{1}{\Omega} \int_{\Omega} \varepsilon(\boldsymbol{x}) \mathrm{d} V$, the following strain decomposition is justified

$$
\boldsymbol{E}=\boldsymbol{E}^{m}+\boldsymbol{E}^{c}
$$

Further, the inelastic deformation induced by a family of microcrakcs of normal $\underline{n}$, noted $\varepsilon^{c}$, takes the general form:

$$
\varepsilon^{c}(\underline{n})=\beta(\underline{n}) \underline{n} \otimes \underline{n}+\underline{\gamma}(\underline{n}) \otimes \underline{n} .
$$

Above, the vector $\underline{\gamma}$ and the scalar $\beta$ represent the relative shear sliding and the averaged opening between cracks lips, respectively. The macroscopic inelastic strain $\boldsymbol{E}^{c}$ is then obtained by taking integration of $\varepsilon^{c}(\underline{n})$ over the surface $\mathcal{S}^{2}$ of a unit sphere:

$$
\boldsymbol{E}^{c}=\frac{1}{\Omega} \int_{\Omega^{c}} \varepsilon^{c} \mathrm{~d} V=\frac{1}{4 \pi} \int_{\mathcal{S}^{2}}(\beta \underline{n} \otimes \underline{n}+\underline{\gamma} \otimes \underline{n}) \mathrm{d} S
$$

Under isotropic consideration, $\boldsymbol{E}^{c}$ is also written explicitly as a sum of a mean part and a deviatoric part:

$$
\boldsymbol{E}^{c}=\frac{1}{3} \beta \boldsymbol{\delta}+\boldsymbol{\Gamma}
$$

with

$$
\beta=\operatorname{tr} \boldsymbol{E}^{c}, \quad \boldsymbol{\Gamma}=\frac{1}{4 \pi} \int_{\mathcal{S}^{2}} \underline{\gamma} \otimes \underline{n} \mathrm{~d} S,
$$

where use has been made of the identity $\frac{1}{4 \pi} \int_{\mathcal{S}^{2}} n_{i} n_{j} \mathrm{~d} S=\frac{1}{3} \delta_{i j}$ with $\boldsymbol{\delta}$ being the second order identity tensor in the three-dimensional setting.

\subsection{Determination of free energy}

The key to develop an elastoplastic damage model consists in determining the Helmholtz free energy of the material. Various studies confirm when cracks are closed and frictional, the total free energy in microcracked media contains two additional parts: the elastic reversible energy contributed by the matrix and the blocked energy due to the rugosity of cracks (e.g., Andrieux et al., 1986; Gambarotta and Lagomarsino, 1993; Pensee et al., 2002; Zhu et al., 2008a) 


$$
\Psi=\frac{1}{2}\left(\boldsymbol{E}-\boldsymbol{E}^{c}\right): \mathbb{C}^{s}:\left(\boldsymbol{E}-\boldsymbol{E}^{c}\right)+\frac{1}{2} \boldsymbol{E}^{c}: \mathbb{C}^{p}: \boldsymbol{E}^{c} .
$$

Remark that the energy $\frac{1}{2} \boldsymbol{E}^{c}: \mathbb{C}^{p}: \boldsymbol{E}^{c}$ is stored in the matrix. It may be cumulated or dissipated progressively during frictional sliding. The fourth rank tensor $\mathbb{C}^{p}$ relating linearly the inelastic strain to the local stress upon microcracks is derived by using problem decomposition and the superposition principle (Zhu et al., 2008a)

$$
\mathbb{C}^{p}=\left[\left(\mathbb{I}-\mathbb{A}^{c}\right)^{-1}: \mathbb{A}^{c}: \mathbb{S}^{s}\right]^{-1}
$$

where $\mathbb{S}^{s}=\left(\mathbb{C}^{s}\right)^{-1}$ is the elastic compliance tensor of the matrix. The solid matrix assumed to be isotropic, this tensor takes the form $\mathbb{C}^{s}=3 k^{s} \mathbb{J}+2 \mu^{s} \mathbb{K}$ with $k^{s}$ and $\mu^{s}$ being the bulk modulus and shear modulus of the matrix, respectively. The components of the fourth order isotropic and deviatoric operators $\mathbb{J}$ and $\mathbb{K}$ is given by

$$
J_{i j k l}=\frac{1}{3} \delta_{i j} \delta_{k l}, \quad K_{i j k l}=\frac{1}{2}\left(\delta_{i k} \delta_{j l}+\delta_{i l} \delta_{j k}\right)-\frac{1}{3} \delta_{i j} \delta_{k l} .
$$

The averaged strain concentration tensor $\mathbb{A}^{c}$ links the strain $\boldsymbol{E}^{c}$ to the macroscopic uniform strain $\boldsymbol{E}$ such that $\boldsymbol{E}^{c}=\mathbb{A}^{c}: \boldsymbol{E}$. Note that the estimate developed by Ponte-Castaneda and Willis (1995) allows to taking into account the effect of spatial distribution of phases by introducing, complementarily to the Eshelby tensor $\mathbb{S}_{e}$, the fourth order tensor $\mathbb{S}_{p}$. According to this scheme, the strain concentration tensor $\mathbb{A}^{c, r}$ for the $r^{\text {th }}$ family of cracks is given as (Ponte-Castaneda and Willis, 1995)

$$
\mathbb{A}^{c, r}=\left(\mathbb{I}-\mathbb{S}_{e}^{r}\right)^{-1}:\left[\mathbb{I}+\sum_{r=1}^{r=N} \varphi^{r} \mathbb{S}_{p}:\left(\mathbb{I}-\mathbb{S}_{e}^{r}\right)^{-1}\right]^{-1},
$$

where $\mathbb{I}=\mathbb{J}+\mathbb{K}$ are the fourth order identity tensor.

For the case of isotropic damage, $\mathbb{A}^{c}=\sum_{r=1}^{r=N} \varphi^{r} \mathbb{A}^{c, r}$ is developed into the form

$$
\mathbb{A}^{c}=\frac{48\left(1-\nu^{2}\right) d}{27(1-2 \nu)+16(1+\nu)^{2} d} \mathbb{J}+\frac{480(1-\nu)(5-\nu) d}{675(2-\nu)+64(5-\nu)(4-5 \nu) d} \mathbb{K},
$$

where $\nu$ is the Poisson's coefficient of the matrix and $d$ is the crack density parameter used as the damage variable. Remark that $d$ is scalar-valued since the hypothesis of isotropic damage distribution is adopted here.

By introduction of (11) into (8), $\mathbb{C}^{p}$ is then recast into the same form as that of $\mathbb{C}^{s}$ :

$$
\mathbb{C}^{p}=3 k^{p} \mathbb{J}+2 \mu^{p} \mathbb{K},
$$

with the blocked bulk and shear moduli

$$
k^{p}=\left(\frac{9(1-2 \nu)}{16\left(1-\nu^{2}\right) d}+\frac{4 \nu-2}{3(1-\nu)}\right) k^{s}, \quad \mu^{p}=\left(\frac{45(2-\nu)}{32(1-\nu)(5-\nu) d}+\frac{5 \nu-7}{15(1-\nu)}\right) \mu^{s} .
$$

Provided the system free energy $\Psi\left(\boldsymbol{E}, \boldsymbol{E}^{c}, d\right)$ in $(7)$, the thermodynamic force associated with the inelastic strain $\boldsymbol{E}^{c}$ is determined by standard derivation of $\Psi$ with respect to $\boldsymbol{E}^{c}$ 


$$
\boldsymbol{\sigma}^{c}=-\frac{\partial \Psi}{\partial \boldsymbol{E}^{c}}=\mathbb{C}^{s}:\left(\boldsymbol{E}-\boldsymbol{E}^{c}\right)-\mathbb{C}^{p}: \boldsymbol{E}^{c}
$$

According to Zhu et al. (2008a), $\boldsymbol{\sigma}^{c}$ corresponds to the local stress upon cracks, which, within the thermodynamic framework, should serve as the driving force of the evolution of inelastic strain $\boldsymbol{E}^{c}$.

\subsection{Opening-closure transition condition}

The mean part $p^{c}=\operatorname{tr} \sigma^{c} / 3$ of the local mean stress acts as being the opening-closure transition indicator for microcracks. To be definite,

- when $\operatorname{tr} \boldsymbol{\sigma}^{c} \geq 0$, all cracks are opened. In this case, we easily establish the explicit relations between the strain $\boldsymbol{E}^{c}$ by microcracks and the macrosopic uniform strain $\boldsymbol{E}$ via the strain concentration tensor $\mathbb{A}^{c}$; - when $\operatorname{tr} \sigma^{c}<0$, all microcracks are closed. The inelastic strain is then the consequence of frictional sliding along crack faces and related directly to the relative displacement (discontinuities) between crack lips. Under such condition, explicit relation between $\boldsymbol{E}^{c}$ and $\boldsymbol{E}$ is no longer obtainable.

In fact, the inelastic strain $\boldsymbol{E}^{c}$ can be viewed as friction-induced plastic strain so as that its evolution will be determined by using a friction criterion formulated at the microscopic scale. This criterion then plays the role of yielding function, just like in the classic theory of plasticity .

Remark that in both open and closed cases, the strain $\boldsymbol{E}^{c}$ by microcracks is related to crack propagation which is characterized by damage evolution. On the one hand, the development of crack opening and frictional sliding may cause damage evolution. On the other hand, damage evolution brings redistribution of stress field, which in turn affects the evolution of the strain $\boldsymbol{E}^{c}$ through the friction criterion. Especially in the closed case, energies may dissipate during damage evolution or frictional sliding, more generally, during their coupling process.

\subsection{Friction criterion}

We adopt here the generalized Coulomb criterion to describe the dissipative friction occurring on microcracks. As the local stress $\boldsymbol{\sigma}^{c}$ is the thermodynamic force associated with the strain $\boldsymbol{E}^{c}$, the friction criterion should be a function of some invariants of $\boldsymbol{\sigma}^{c}$ and formulated at microscopic scale. Note that the macroscopic stress-strain relation can be derived from the free energy (7) within the irreversible thermodynamic framework

$$
\boldsymbol{\Sigma}=\frac{\partial \Psi}{\partial \boldsymbol{E}}=\mathbb{C}^{s}:\left(\boldsymbol{E}-\boldsymbol{E}^{c}\right)
$$

The insertion of (15) into (14) yields the relation

$$
\boldsymbol{\sigma}^{c}=\boldsymbol{\Sigma}-\mathbb{C}^{p}: \boldsymbol{E}^{c} .
$$


Next, we perform a two-part decomposition on $\boldsymbol{\sigma}^{c}$. Combination of (16) with (4) and (12) allows to rewritting $\boldsymbol{\sigma}^{c}$ in the form

$$
\boldsymbol{\sigma}^{c}=p^{c} \boldsymbol{\delta}+\boldsymbol{S}^{c}
$$

with $p^{c}=\frac{1}{3} \operatorname{tr} \boldsymbol{\sigma}^{c}=p-k^{b} \beta$ and $\boldsymbol{S}^{c}=\boldsymbol{S}-2 \mu^{b} \boldsymbol{\Gamma}$ where $p$ and $\boldsymbol{S}$ are the mean part and the deviatoric part of the macroscopic stress tensor $\boldsymbol{\Sigma}$, respectively.

The Coulomb friction criterion is thus a function of $p^{c}$ and $\boldsymbol{S}^{c}$ and takes the usual form

$$
F=\left\|\boldsymbol{S}^{c}\right\|+\tilde{c}_{f} p^{c} \leq 0
$$

where $\tilde{c}_{f}$ is the current effective friction coefficient, which, in view of asperities on crack faces, is not a constant but dependent of the local confining pressure $p^{c}$. The theoretical determination of the relationship between $\tilde{c}_{f}$ and the pressure $p^{c}$ as well as the matrix intrinsic friction coefficient is quite difficult, mainly due to the fact that cracks surfaces are generally complex in geometry and evolving (degraded) during the cumulation of frictional sliding. For simplicity, we propose the following empirical expression, which is based on laboratory observations for conventional triaxial compression tests:

$$
\tilde{c}_{f}=\left(1-\frac{\left\langle p_{\text {ref }}-p^{c}\right\rangle}{p_{\text {ref }}}\right)^{m} c_{f},
$$

where the operator $\langle x\rangle$ is such that $\langle x\rangle=\frac{x+|x|}{2}, p_{\text {ref }}$ serves as a reference confining pressure, $m$ is a parameter used to control the evolution and $c_{f}$ is interpreted as the natural friction coefficient in its perfectly compact state. Thus, the physical significance of (19) consists in taking into consideration the compacting effect of compression on the friction coefficient. Precisely, when $p^{c}$ is less than $p_{\text {ref }}$, the nominal (effective) friction coefficient $\tilde{c}_{f}$ is smaller than the value $c_{f} ; \tilde{c}_{f}$ will increase as $p^{c}$ becomes larger. This implies that as $p^{c}$ increases, crack faces are getting more compressed and the occlusion between crack faces becomes tighter, which increases the contact area between crack faces and consequently the effective friction coefficient $\tilde{c}_{f}$ increases. When $p^{c}$ approaches $p_{r e f}$, the crack closure occurs at its critical state where the effective friction coefficient $\tilde{c}_{f}$ equals to the friction coefficient $c_{f}$ and the crack is considered completely compacted. Therefore, we can take $p_{\text {ref }}$ as the stress that makes microcracks completely occluded. It is worth noting that the value of $p_{\text {ref }}$ should not be too large, because excessive compression will cause some crash of asperities on crack surfaces, which in turn decreases the friction coefficient.

The friction criterion (18) can be rewritten in the following more explicit form

$$
F=\left\|\boldsymbol{S}-2 \mu^{b} \boldsymbol{\Gamma}\right\|+\tilde{c}_{f}\left(p-k^{b} \beta\right) \leq 0 .
$$

Above, in the framework of plasticity theory, $2 \mu^{b} \boldsymbol{\Gamma}$ and $k^{b} \beta$ provide kinematic hardening/softening and isotropic hardening/softening on yielding surface, respectively. In this sense, $\left(\mathbb{C}^{p}: \boldsymbol{E}^{c}\right)$ appeared in (16) plays a role of back stress. Remark that in widely used phenomenological, modelling of material plastic hardening/softening is usually carried out by introduction of complementary laws. 


\subsection{Potential function}

It is more appropriate for geomaterials to use a non-associated flow rule to describe volumetric deformation. To this end, the following plastic potential function is proposed:

$$
G=\left\|\boldsymbol{S}^{c}\right\|+c_{f} p^{c}=0
$$

To be consistent, this function $G$ is similar to that of the friction criterion $F$. The evolution rate of the plastic strain $\boldsymbol{E}^{c}$ is determined by following the normality rule:

$$
\dot{\boldsymbol{E}}^{c}=\dot{\lambda}^{f} \frac{\partial G}{\partial \boldsymbol{\sigma}^{c}}=\dot{\lambda}^{f}\left(\boldsymbol{V}+\frac{1}{3} c_{f} \boldsymbol{\delta}\right)
$$

It follows the evolution rate of the variables $\beta$ and $\Gamma$ :

$$
\dot{\beta}=\dot{\lambda}^{f} c_{f} ; \dot{\boldsymbol{\Gamma}}=\dot{\lambda}^{f} \boldsymbol{V}
$$

where $\boldsymbol{V}=\boldsymbol{S}^{c} /\left\|\boldsymbol{S}^{c}\right\|$ denotes the sliding direction, and $\dot{\lambda}^{f}$ is a non-negative multiplier which can be calculated by using the consistency condition $(F=0, \dot{F}=0)$.

\subsection{Damage criterion}

Within the framework of irreversible thermodynamics, damage criterion should be a function of the thermodynamic force that conjugates with damage variable $d$. The damage conjugate force $F^{d}$ is derived from the total free energy (7):

$$
F^{d}=-\frac{\partial \Psi}{\partial d}=-\frac{1}{2} \boldsymbol{E}^{c}: \frac{\partial \mathbb{C}^{p}}{\partial d}: \boldsymbol{E}^{c}
$$

By combining (4) and (12), the above formulation can be recast into the form:

$$
F^{d}=\frac{9(1-2 \nu)}{32\left(1-\nu^{2}\right) d^{2}} k^{s} \beta^{2}+\frac{45(2-\nu)}{64(1-\nu)(5-\nu) d^{2}} \mu^{s} \boldsymbol{\Gamma}: \boldsymbol{\Gamma} .
$$

The following damage criterion in exponential form is used:

$$
f_{d}=d_{c}-\left(d_{c}-d_{0}\right)\left[\exp \left(-c_{1} F^{d}\right)\right]-d \leq 0 .
$$

where $d_{0}$ and $d_{c}$ are respectively the initial and critical value of damage variable, and the model parameter $c_{1}$ controls damage hardening. Particularly, it is seen from (12) that damage is directly related to the stored energy via the plastic blocked stiffness $\mathbb{C}^{p}$. Inversely, the induced damage causes degradation of $\mathbb{C}^{p}$ and further influences the evolution of inelastic deformation. From physical point of view, it is possible to derive $d_{c}$ from the condition $\mu^{p} \geq 0$, which yields

$$
d_{c}=\frac{675(2-\nu)}{32(5-\nu)(7-5 \nu)} .
$$

Finally, suppose also that damage evolution rate obeys the normality rule, i.e.

$$
\dot{d}=\dot{\lambda}^{d} \frac{\partial f_{d}}{\partial F^{d}}
$$




\subsection{Coupling between friction and damage}

It is seen from (24) that $F^{d}$ is only dependent of the blocked energy by microcracks, that is to say, damage evolution is totally driven by frictional sliding along microcrack surfaces. On the other hand, as shown in (14) and (16), damage propagation can bring redistribution of the stress via the term $\left(\mathbb{C}^{p}: \boldsymbol{E}^{c}\right)$, which in turn influences the progress of frictional sliding. In fact, the Coulomb-type criterion $F$ is also implicitly function of the damage variable $d$ through $\mu^{b}$ and $k^{b}$. Therefore, damage and friction are generally strongly coupled. Remark that compared to phenomenological models, the present coupling formulation between plasticity and damage seems more natural and consistent without introduction of other laws.

To determine the multipliers $\dot{\lambda}^{d}$ and $\dot{\lambda}^{f}$, one has to take into account both the damage and friction criteria because of their potential coupling. When they are saturated at the same time, the combination of consistency conditions corresponding to these two criteria gives

$$
\left\{\begin{array}{l}
\dot{G}=\frac{\partial G}{\partial d} \dot{d}+\frac{\partial G}{\partial \beta} \dot{\beta}+\frac{\partial G}{\partial \boldsymbol{\Gamma}}: \dot{\boldsymbol{\Gamma}}+\frac{\partial G}{\partial \boldsymbol{E}}: \dot{\boldsymbol{E}}=0 \\
\dot{f}_{d}=\frac{\partial f_{d}}{\partial d} \dot{d}+\frac{\partial f_{d}}{\partial \beta} \dot{\beta}+\frac{\partial f_{d}}{\partial \boldsymbol{\Gamma}}: \dot{\boldsymbol{\Gamma}}=0
\end{array}\right.
$$

whose resolution leads finally to the multipliers $\dot{\lambda}^{f}$ and $\dot{\lambda}^{d}$

$$
\left\{\begin{array}{l}
\dot{\lambda}^{f}=\frac{1}{H} \frac{\partial F}{\partial \boldsymbol{E}}: \dot{\boldsymbol{E}} \\
\dot{\lambda}^{d}=-\frac{\frac{\partial f_{d}}{\partial \beta} c_{f}+\frac{\partial f_{d}}{\partial \boldsymbol{\Gamma}}: \boldsymbol{V}}{\frac{\partial f_{d}}{\partial d} H} \frac{\partial F}{\partial \boldsymbol{E}}: \dot{\boldsymbol{E}}
\end{array}\right.
$$

with $H=\frac{\partial F}{\partial d}\left(\frac{\partial f_{d}}{\partial \beta} c_{f}+\frac{\partial f_{d}}{\partial \boldsymbol{\Gamma}}: \boldsymbol{V}\right) / \frac{\partial f_{d}}{\partial d}-\left(\frac{\partial F}{\partial \beta} c_{f}+\frac{\partial F}{\partial \boldsymbol{\Gamma}}: \boldsymbol{V}\right)$.

\section{Numerical simulations}

In this part, the developed micromechanics-based elastoplastic damage model is applied to describe experimental triaxial compression data from diabase samples at two weathering levels.

\subsection{Parameters and computational algorithm}

The proposed micromechanics-based model is now applied to simulate the triaxial compression tests. The key feature of this isotropic model consists in taking into consideration the coupling between damage and friction (friction-induced plasticity) at the microscopic scale. When comparing with phenomenological models, the number of model parameters has been significantly reduced. In total, there are seven parameters to be determined: two elastic constants $E$ and $\nu$ which can be obtained from the elastic phase of the stress-strain curves; the friction coefficient $c_{f}$ which can be identified from the envelope of peak 
strengths under different confining pressures; $p_{r e f}$ and $m$ control the variation of the effective friction coefficient; damage parameters $d_{0}$ denotes initial state of damage and $c_{1}$ controls the damage evolution rate. When lack of experimental measure, one can take a small value for $d_{0}$ for material in its initial state.

The proposed model is numerically implemented in a computer code by using the operator-split algorithm, which contains an elastic predictor and a plastic-damage corrector. Since damage is totally induced by the friction-induced plastic deformation, it is convenient to perform plastic iteration and damage iteration in sequence, i.e. damage corrector can be performed after plastic corrector. The general procedure goes in the following sequences as shown in Fig. 3.

\subsection{Simulation of fresh diabase rock samples}

The aforementioned model constants and parameters for two sets of diabase samples are listed in table 2. Comparisons between simulation results and experimental data under different confining pressures are shown in Fig. 4. It is seen that the proposed model can describe the brittle features. A good agreement is recorded between the simulation results and experimental data under all confining pressures, both in axial and lateral strains.

\subsection{Simulation of slightly weathered diabase rock samples}

In this section, two methods are used to simulate the mechanical behavior of slightly weathered rock samples. The purpose is to verify the applicability of the proposed model to slightly weathered rocks and reveal the effect of weathering on mechanical properties of rocks at the same time.

- Method 1: The first method consists in treating the weathered rock as a different material. All parameters involved in the model have been determined from experimental data (see Table 2). Compared with the fresh rock samples, the elastic modulus $E$ as well as the peak strengths of the slightly weathered rock under different confining pressures decrease significantly. The mechanical behavior for slightly weathers rock samples are simulated and the numerical results are shown in Fig. 5.

Table 2

Collections of parameters for numerical modelling

\begin{tabular}{cccccccc}
\hline \hline Samples & $E(\mathrm{MPa})$ & $\nu$ & $c_{f}^{\prime}$ & $c_{1}$ & $d_{0}$ & $p_{\text {ref }}$ & $m$ \\
\hline Fresh diabase & 52 & 0.18 & 1.0 & 0.8 & 0.001 & 150 & 0.8 \\
weathered (method 1) & 40 & 0.18 & 0.8 & 0.6 & 0.001 & 150 & 0.8 \\
weathered (method 2) & 52 & 0.18 & 0.9 & 0.55 & 0.2 & 150 & 0.8 \\
\hline
\end{tabular}


- Method 2: The main impact of weathering on the mechanical properties of rocks is the increase in porosity, as we can see in Table 1. Especially in slightly weathered state, the cement structure of the material is almost unchanged. From mechanical viewpoint, it is reasonable to relate the increase in porosity to the initial damage state before loading. That is to say, there exist a priori microcracks induced by weathering process, which leads to some deterioration in mechanical behaviour. Therefore, instead of redetermining the material constants and model parameters from mechanical tests on weathered rock samples, we can use an initial damage value to describe the material degradation due to weathering. We adopt here $d_{0}=0.2$. One has

$$
E=\left(1-d_{0}\right) E_{0}, \quad c_{f}=\left(1-d_{0}\right) c_{f 0}
$$

where $E_{0}$ and $c_{f 0}$ correspond to the measures for fresh rocks. With these parameters, simulation results under different confining pressures are shown in Fig. 6.

It is seen from Fig. 5 that the mechanical behaviour of weathered rock can be predicted by adjusting the initial damage value and damage evolution rate (parameter $c_{1}$ ), with elastic constants $E$ and $\nu$ identical to those for fresh rock. A satisfactory agreement is reported between simulation results and testing data, although room still exists to improve the method 2. For example, a large number of experimental data for different weathering levels is required to establish the relation between porosity and initial damage.

\section{Conclusions}

A micromechanics-based elastoplastic damage model is developed for the description of mechanical behaviors of quasi brittle rocks. This model takes into account coupling between frictional sliding and damage evolution as well as unilateral effects. The model is formulated in a proper framework of dissipative thermodynamics by considering local physical mechanisms of inelastic behaviors. The proposed Coulombtype friction criterion takes into account the effect of local confining on the effective friction coefficient. The proposed model contains a relatively small number of parameters whose physical meanings are clearly identified. Numerical simulations of laboratory tests on two series of rock samples have shown that the proposed model is able to describe the main features of mechanical responses of quasi brittle rocks. However, for rock materials in weathered conditions or with higher porosity, plastic deformation of rock matrix before failure becomes an important mechanism. This suggests an extension of the present work consisting in accounting for inelastic deformation in rock matrix coupled with microcrack growth. Finally, another future work will be considered on the hydromechanical coupling in porous rocks exhibiting plastic deformation and damage evolution. 


\section{Acknowledgements:}

The authors are grateful for the financial support by National Natural Science Foundation of China under grant number 50778138. Ni XIE is grateful to China Scholarship Council for providing her a scholarship during her stay in France.

\section{References}

Andrieux, S., Bamberger, Y., Marigo, J., 1986. Un modèle de matériau microfissuré pour les roches et les bétons. Journal de mécanique théorique et appliquée 5(3), 471-513.

Bozkurtoglu, E., Vardar, M., Suner, F., Zambak, C., 2006. A new numerical approach to weathering and alteration in rock using a pilot area in the tuzla geothermal area, turkey. Engineering Geology 87, 33-47.

Brace, W., Bombolakis, E., 1963. A note on brittle crack growth in compression. Journal of Geophysical Research 68(12), 3709-3713.

Budiansky, B., O’Connel, J., 1976. Elastic moduli of a cracked solid. Int. J. Solids Structures 12, 81-97.

Chaboche, J.-L., P., K., 2005. On the capabilities of mean-field approaches for the description of plasticity in metal matrix composites. Int. Journ. of Plasticity 21(7), 1409-1434.

Chow, C., June, W., 1987. An anisotropic theory of elasticity for continuum damage mechanics. Int J Fract 33, 3-16.

Dormieux, L., Kondo, D., Ulm, F.-J., 2006. Microporomechanics. WILEY.

Gambarotta, L., Lagomarsino, S., 1993. A microcrak damage model for brittle materials. Int. J. Solids Structures 30(2), 177-198.

Halm, D., Dragon, A., 1996. A model of anisotropic damage by mesocrack growth: unilateral effect. Int J Damage Mech 5, 384-402.

Horii, H., Nemat-Nasser, S., 1985. Compression-induced microcrack growth in brittle solids: axial splitting and shear failure. Journal of Geophysical Research 90(B4), 3105-3125.

Ju, J., 1989. On energy based coupled elastoplastic damage theories: constitutive modeling and computational aspects. Int J Fract 25(7), 803-833.

Kranz, R., 1983. Microcracks in rocks: a review. Tectonophysics 100, 449-480.

Murakami, S., Kamiya, K., 1996. Constitutive and damage evolution equations of elastic brittle materials based on irreversible thermodynamics. Int J Mech Sci 39(4), 473-486.

Pensee, V., Kondo, D., Dormieux, L., 2002. Micromechanical analysis of anisotropic damage in brittle materials. J. Engng. Mech., ASCE 128(8), 889-897.

Ponte-Castaneda, P., Willis, J.-R., 1995. The effect of spatial distribution of effective behavior of composite 
materials and cracked media. Journ. Mech. Phys. Solids 43, 1919-1951.

Tugrul, A., 2004. The effect of weathering on pore geometry and compressive strength of selected rock types from turkey. Engineering Geology 75, 215-227.

Wawersik, W., Brace, W., 1971. Post-failure behavior of a granite and diabase. Rock Mech 3, 61-85.

Wong, T., 1982. Micromechanics of faulting in westerly granite. Int J Rock Mech Min Sci 19, 49-56.

Zhu, Q.-Z., Kondo, D., Shao, J.-F., 2008a. Micromechanical analysis of coupling between anisotropic damage and friction in quasi brittle materials: role of the homogenization scheme. International Journal of Solids and Structures 45(5), 1385-1405.

Zhu, Q.-Z., Kondo, D., Shao, J.-F., Pensee, V., 2008b. Micromechanical modelling of anisotropic damage in brittle rocks and application. International Journal of Rock Mechanics and Mining Sciences 45, 467-477.

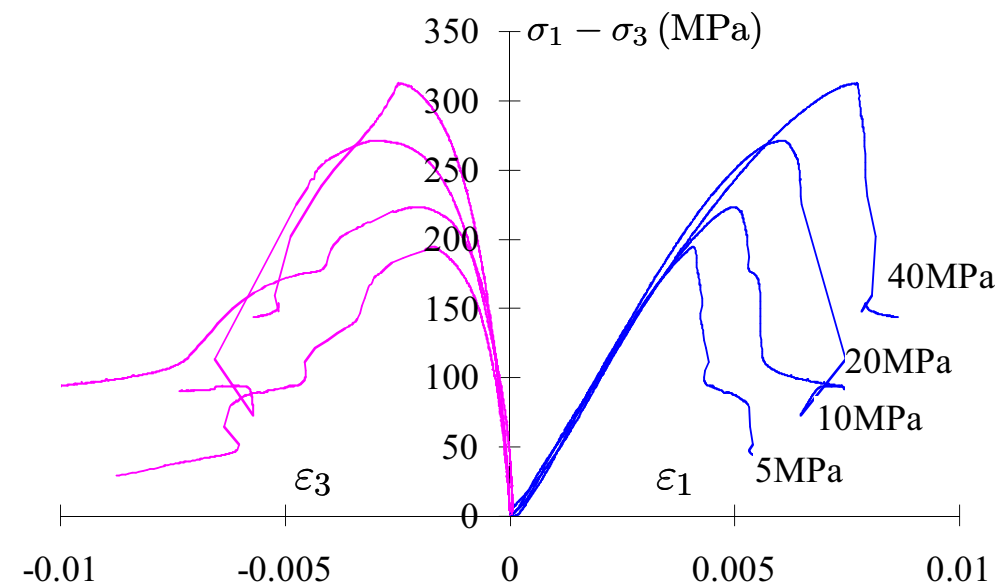

Figure 1. Stress-strain curves in triaxial compression tests for fresh diabase rock samples

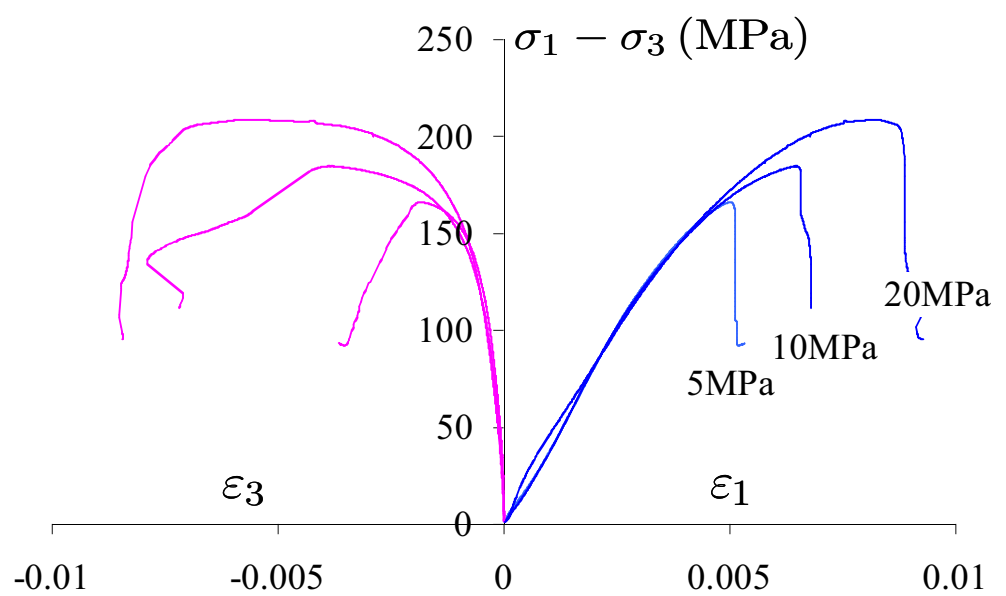

Figure 2. Stress-strain curves in triaxial compression tests for slightly weathered diabase rock samples 


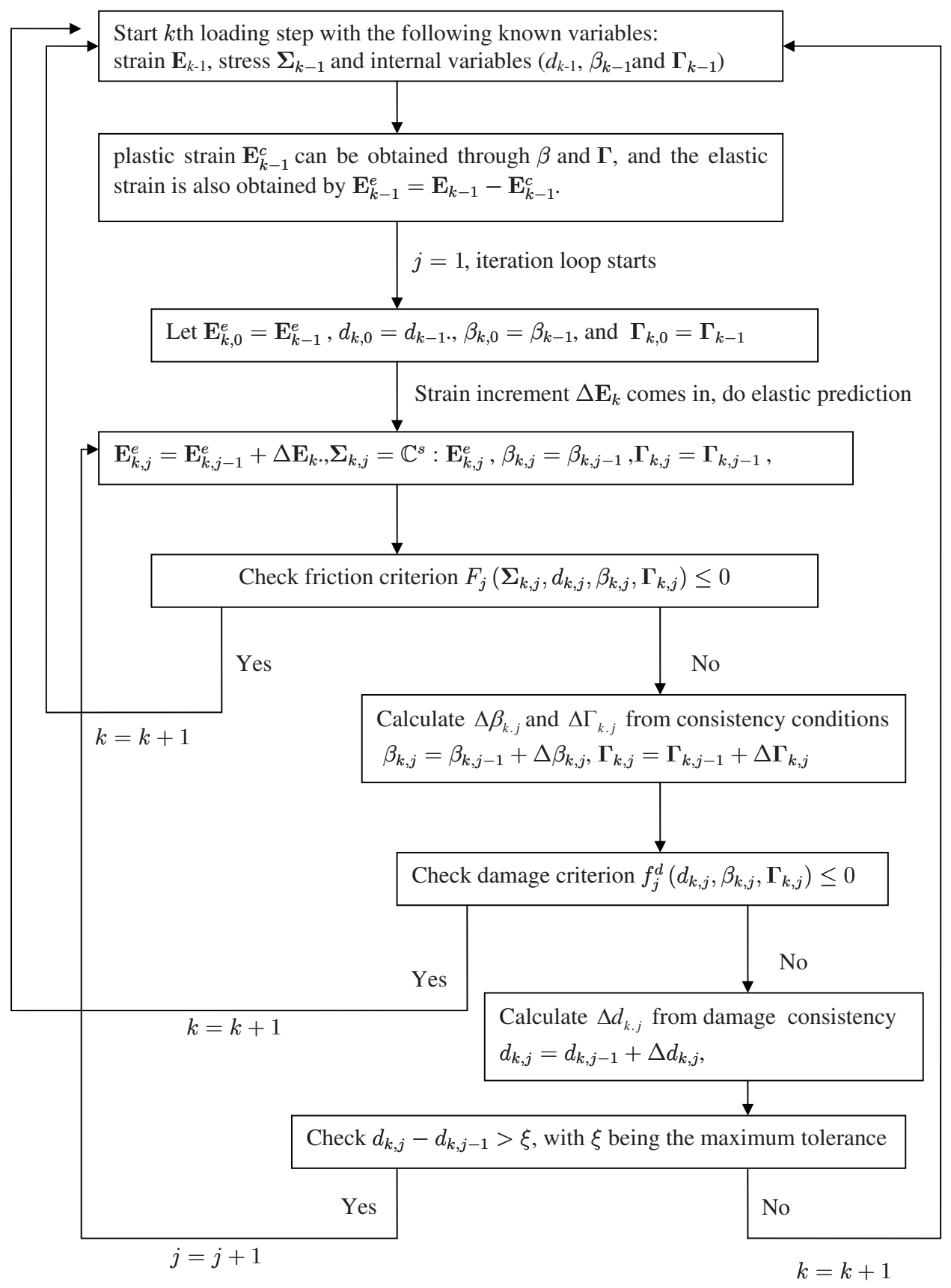

Figure 3. Computational iteration algorithm 

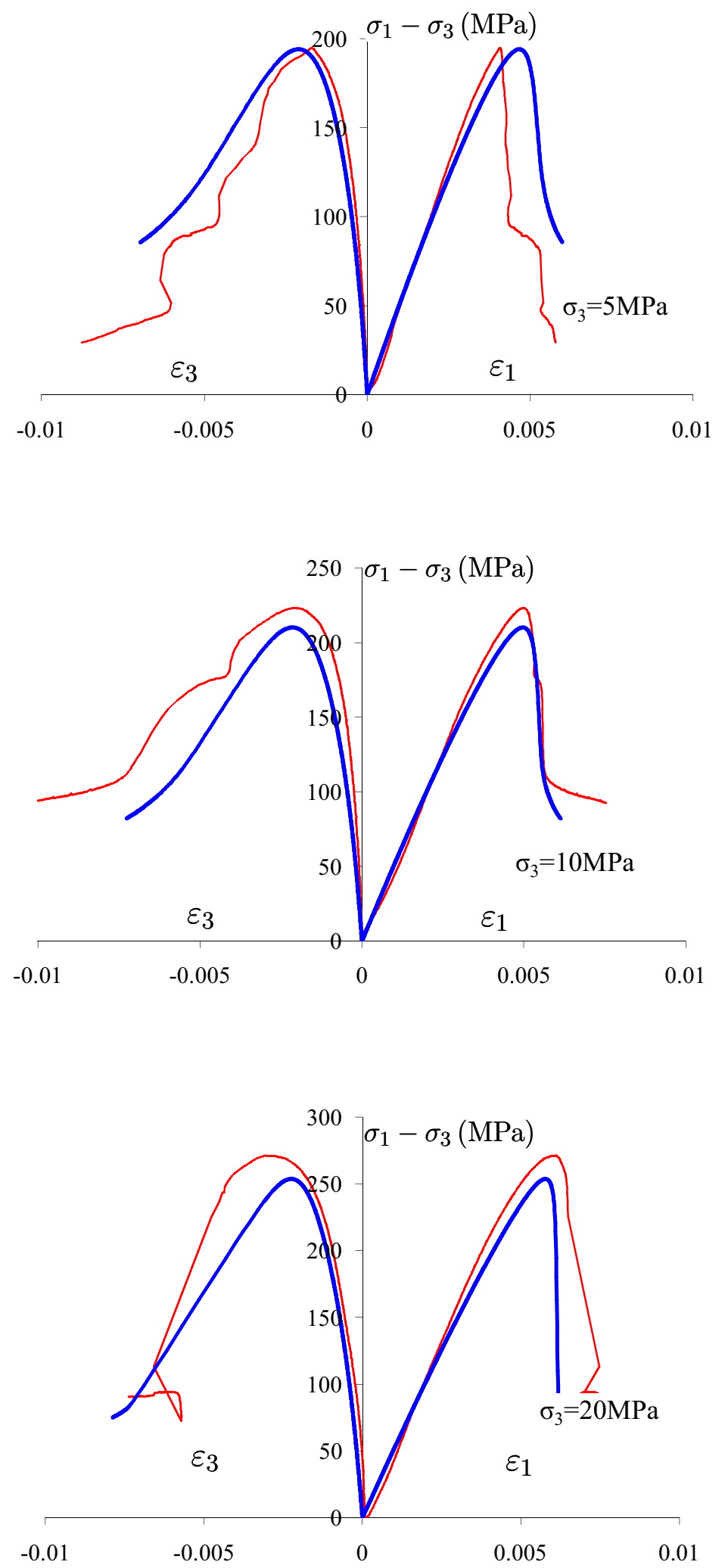


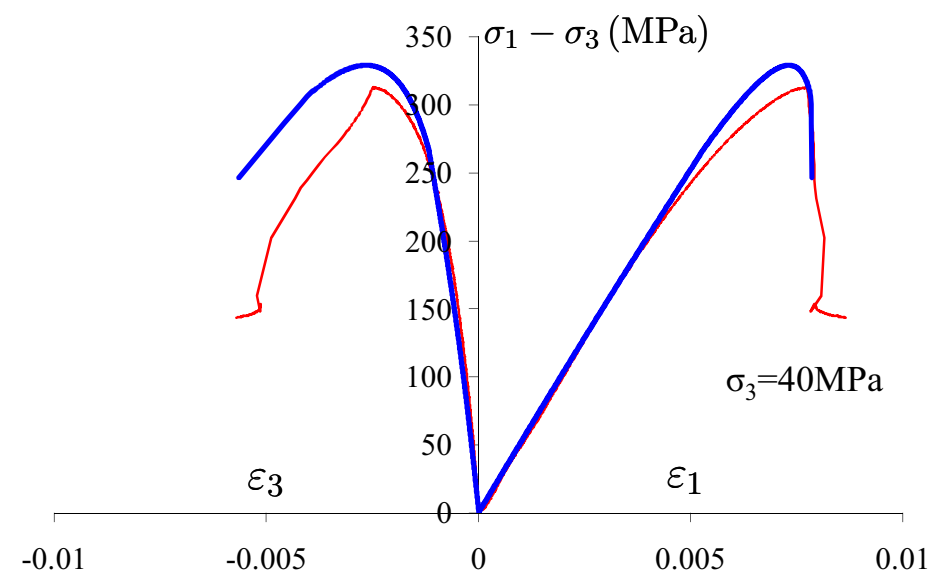

Figure 4. Comparisons between model predictions (thick lines) and experimental data (thin lines) under different confining pressures (fresh samples, $P_{c}=5,10,20$ and $40 \mathrm{MPa}$ )
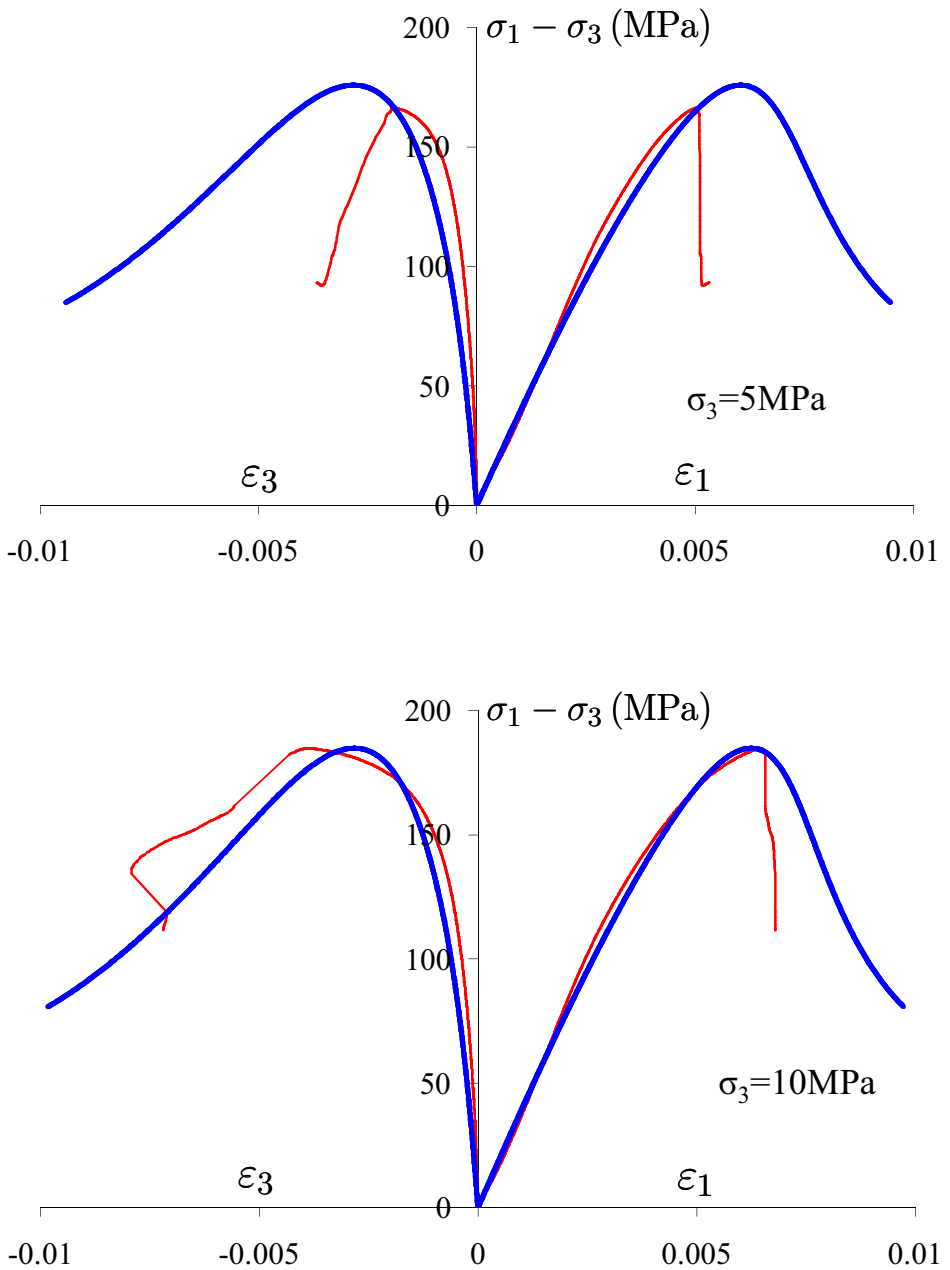


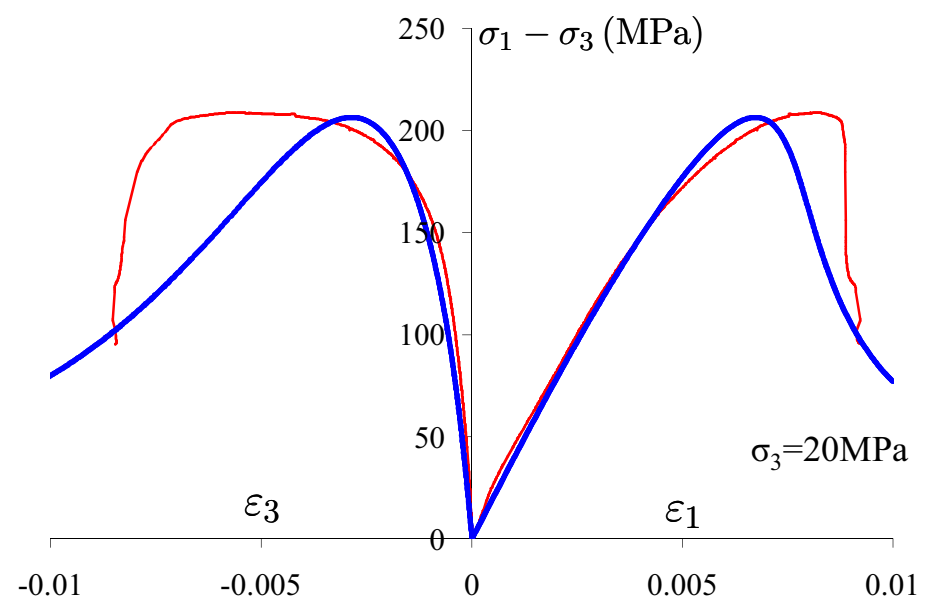

Figure 5. Comparisons between model predictions (thick lines) and experimental data (thin lines) under different confining pressures (slightly weathered samples, $P_{c}=5,10,20 \mathrm{MPa}$ ): method 1
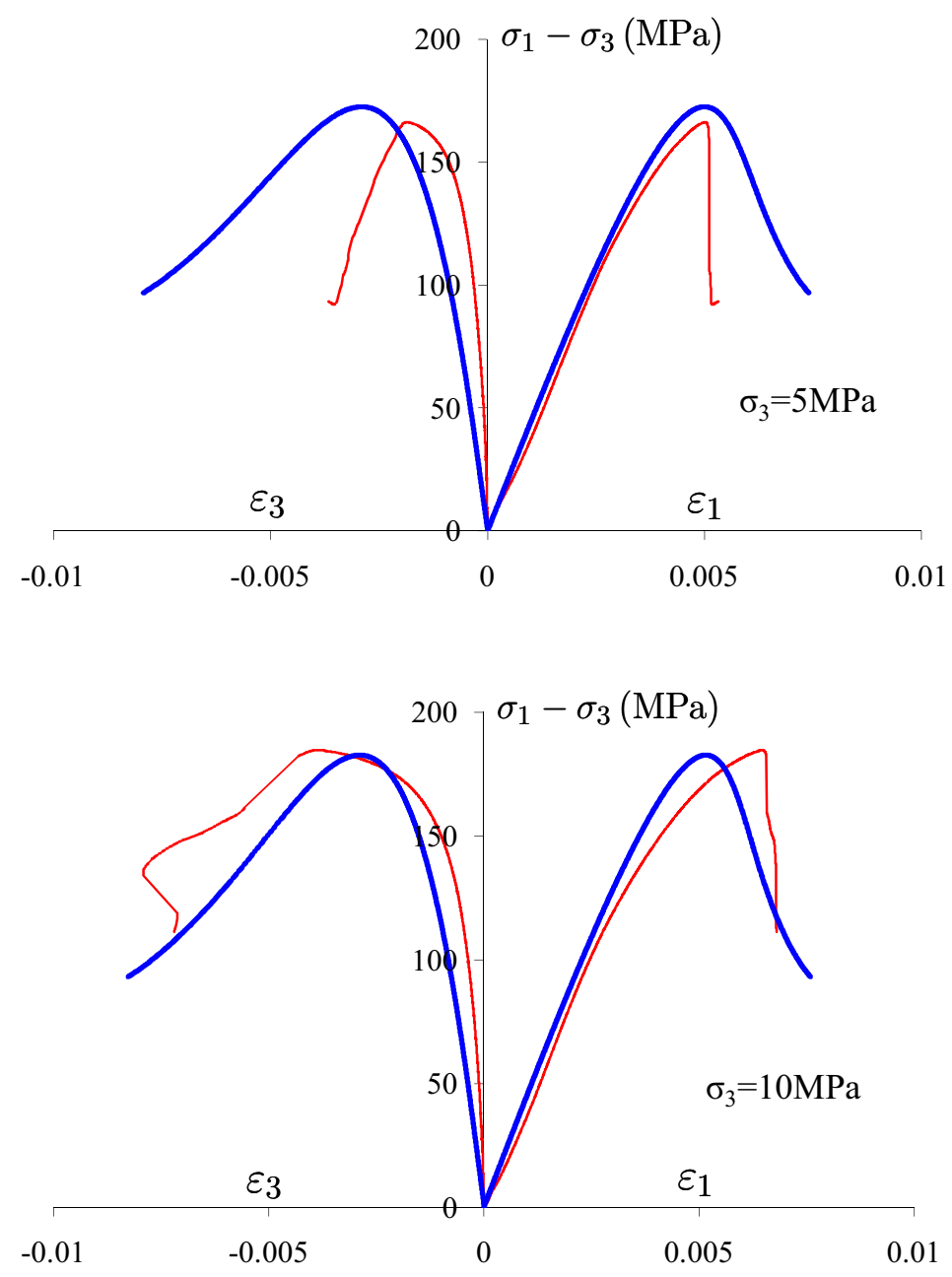


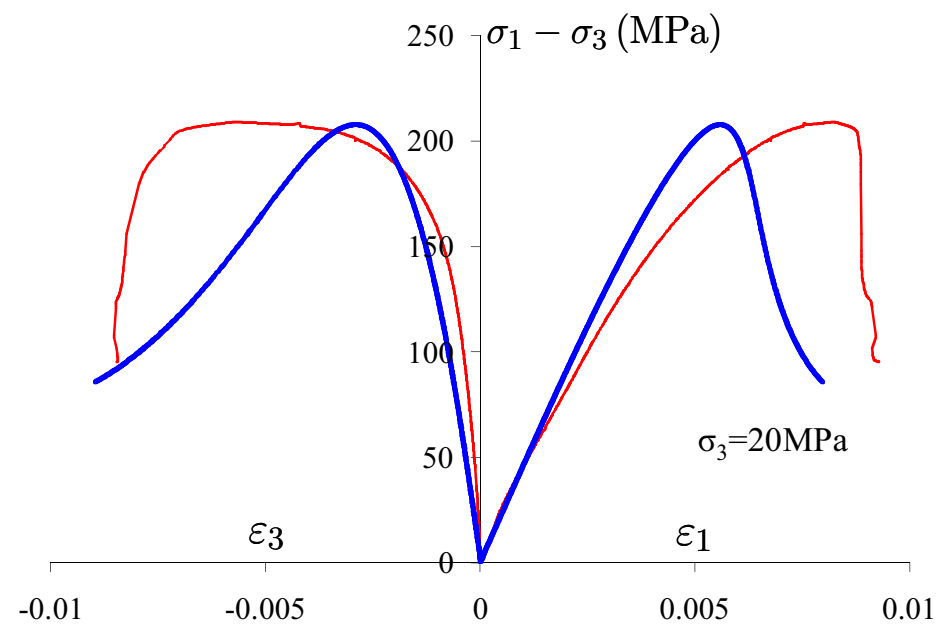

Figure 6. Comparisons between model predictions (thick lines) and experimental data (thin lines) under different confining pressures (slightly weathered samples, $P_{c}=5,10,20 \mathrm{MPa}$ ): method 2 